\title{
One-Dimensionally Polarization-Independent Retrodirective Metasurface
}

\author{
Jae-Gon Lee ${ }^{1} \cdot$ Jeong-Hae Lee ${ }^{2, *}$
}

\begin{abstract}
A one-dimensionally polarization-independent retrodirective metasurface (PIRMS) is proposed in this letter. The retrodirective metasurface (RMS) consists of ring structures on its ground plane to achieve the polarization-independent characteristics. The reflection phases of unitcells of the metasurface can be controlled by the radius of the ring structure, and the dimension of the supercell with the $2 \pi$ phase variation depends on an incident angle and operation frequency from the generalized Snell's law. To verify its feasibility, two kinds of RMSs to operate at two retrodirected angles of $20^{\circ}$ and $40^{\circ}$ are designed and measured at $5.8 \mathrm{GHz}$. The measured retroreflected power efficiencies are more than $94 \%$ and $92 \%$ at the retrodirected angles of $20^{\circ}$ and $40^{\circ}$, respectively, regardless of the polarization. The results show that the proposed RMS has good performances independent of polarization.
\end{abstract}

Key Words: Generalized Snell's Law, Polarization, Retrodirective Metasurface.

\section{INTRODUCTION}

Since metasurfaces (MSs) can manipulate the wave-front of transmitted and reflected electromagnetic (EM) waves, they have a wide range of applications in EM field. In 2011, the phase gradient MS, which can manipulate the wave-front of anomalous reflection and refraction waves, is proposed by $\mathrm{Yu}$ et al. [1]. In [1], a generalized Snell's law is described from Fermat's principle. Since then, there has been extensive research related to anomalous reflection [2-4]. Specifically, retrodirectivity can be achieved using the planar type of a MS using the generalized Snell's law [5, 6]. In [5], a MS simultaneously operating at multiple incident angles designed using array of striplines on the ground plane for only the TM wave. Additionally, binary MS was observed to achieve an efficient retroreflector for each TE and TM wave at near-grazing incidence [6].
In this letter, a one-dimensionally polarization-independent retrodirective metasurface (PIRMS), which consists of several supercells with a continuous gradient phase of $2 \pi$, is proposed. The length of a supercell depends on its specific retrodirective angle and its wavelength of an operation frequency calculated by the generalized Snell's law. Also, the unitcell of a supercell is composed of a ring structure on the ground plane that simultaneously operates the TE and TM mode. The reflection phases of these unitcells can be controlled by the radius and width of the ring structure. Moreover, the performance of the proposed PIRMS is confirmed by a theoretical analysis, a full-wave simulation, and a measurement at two retrodirected angles of $20^{\circ}$ and $40^{\circ}$.

\section{DESIGN OF 1D POLARIZATION-INDEPENDENT}

Manuscript received May 3, 2019 ; Revised July 4, 2019 ; Accepted August 8, 2019. (ID No. 20190503-034J)

${ }^{1}$ Department of Electronic Engineering, Kyungnam University, Changwon, Korea.

${ }^{2}$ School of Electronic and Electrical Engineering, Hongik University, Seoul, Korea.

"Corresponding Author: Jeong-Hae Lee (e-mail: jeonglee@hongik.ac.kr)

This is an Open-Access article distributed under the terms of the Creative Commons Attribution Non-Commercial License (http://creativecommons.org/licenses/by-nc/4.0) which permits unrestricted non-commercial use, distribution, and reproduction in any medium, provided the original work is properly cited.

(c) Copyright The Korean Institute of Electromagnetic Engineering and Science. 


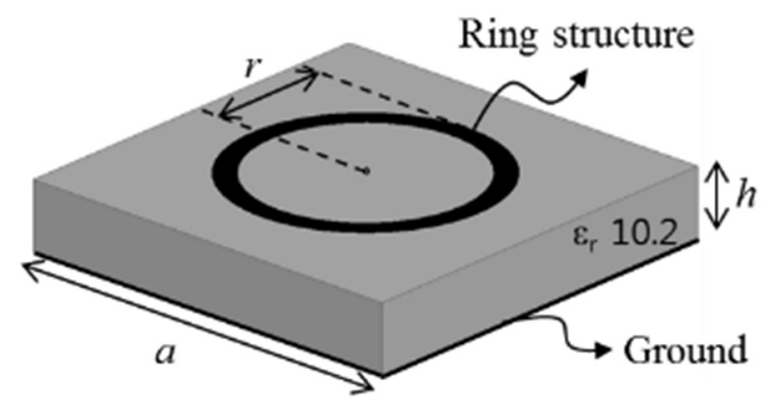

Fig. 1. Unitcell of the proposed $1 \mathrm{D}$ polarization-independent retrodirective metasurface (PIRMS).

Based on the generalized Snell's law, MS with a $2 \pi$ phase gradient contributes to the generation of reflected and transmitted anomalous waves [1]. From the generalized Snell's law of reflection, the relation between the incident angle $\theta_{i}$ and the anomalous reflection angle $\theta_{r}$ along the interface $\left(d \Phi_{y} / d y\right)$ can be defined as [1]:

$$
\sin \theta_{r}-\sin \theta_{i}=\frac{\lambda_{0}}{2 \pi n_{i}} \frac{d \Phi_{y}}{d y}=\frac{\lambda_{0}}{2 \pi n_{i}} \frac{2 \pi}{L_{y}}=\frac{\lambda_{0}}{n_{i} L_{y}}
$$

where $\lambda_{0}$ and $n_{i}$ represent the wavelength in free space and the refractive index of the incident medium, respectively. $d \Phi y / d y$ indicates the phase gradient of reflection along $\mathrm{y}$-direction. To achieve retroreflection $\left(\theta_{r}=-\theta_{i}\right)$, the length of a supercell $(L y)$ with a $2 \pi$ phase gradient can be expressed as from Eq. (2):

$$
L_{y}=\frac{\lambda_{0}}{2 n_{i} \sin \theta_{r}}
$$

Moreover, the supercell is composed of a series of unitcells that have characteristics of a high reflectivity and a reflection phase variation of $2 \pi$ at the operation frequency. Fig. 1 shows the unitcell of the proposed 1D PIRMS. To obtain polarization-independent reflection characteristics, a structurally symmetrical ring structure on the ground plane is employed in this letter. Also, the ring structure can be designed more compact rather than a circular structure with a symmetrical shape. The operation frequency is $5.8 \mathrm{GHz}$ and the utilized substrate is RT Duroid $6010\left(\varepsilon_{r}=10.2, h=3.175 \mathrm{~mm}\right.$, and $\left.\tan \delta=0.0023\right)$. The width of the ring structure is fixed to be $0.5 \mathrm{~mm}$, considering manufacturing tolerance. When the thickness of a substrate is greater, the slope of the reflection phase becomes gentler. If the width of the ring structure becomes narrower, the frequency with the reflection phase of $0^{\circ}$ down-shifts. The lengths of the supercells can be calculated as $75.6 \mathrm{~mm}$ and $40 \mathrm{~mm}$ from Eq. (2) in the cases of retrodirected angle of $20^{\circ}$ and $40^{\circ}$, respectively. Thus, the supercell consists of six unitcells with a dimension of $12.6 \mathrm{~mm}\left(0.24 \lambda_{0}\right)$ in the case of retrodirected angle of $20^{\circ}$, as shown in Fig. 2(a). Similarly, the supercell consists of four unitcells with a dimension of $10 \mathrm{~mm}\left(0.19 \lambda_{0}\right)$ in the case of a retrodirected angle of $40^{\circ}$, as shown in Fig. 2(b). Fig. 3 shows the full-wave simulated reflection response of the ring structure against the radius $(r)$ in both the retrodirected angle of $20^{\circ}$ and $40^{\circ}$ at $5.8 \mathrm{GHz}$. When the substrate is thinned, the reflection phase against the size of the unitcell changes sharply. Then, the sizes of the unitcells composed of the supercell become almost the same. If the manufacturing method is not precise, the reflection phase variation of $2 \pi$ cannot be measured. Therefore, it is advantage to use a thick substrate to obtain retrodirectivity. The reflection response of the unitcell is analyzed using the masterslave periodic condition and Floquet port in ANSYS's Electronics desktop software. The reflection magnitude remains high $(>0.98)$, as the geometrical parameter $r$ varies from $0.5 \mathrm{~mm}$ to 6 $\mathrm{mm}$, as shown in Fig. 3. Also, the reflection phase range of $2 \pi$ can be obtained by changing the radius.

\section{MEASURED RESULTS AND DISCUSSION}

To verify the retrodirectivity, the 1 D PIRMSs with $29 \times 6$ $(365.4 \mathrm{~mm} \times 453.6 \mathrm{~mm})$ and $38 \times 11(370 \mathrm{~mm} \times 520 \mathrm{~mm})$ supercells were designed and fabricated for retrodirected angle of $20^{\circ}$ and $40^{\circ}$, respectively. The reflected power can be meas-

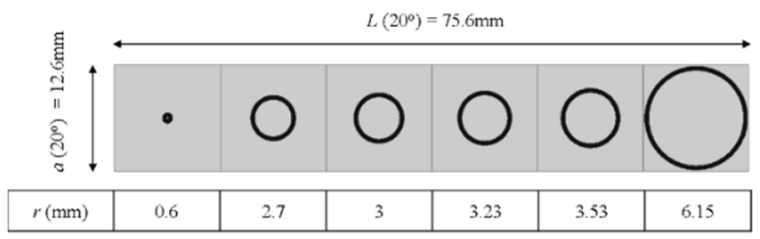

(a)

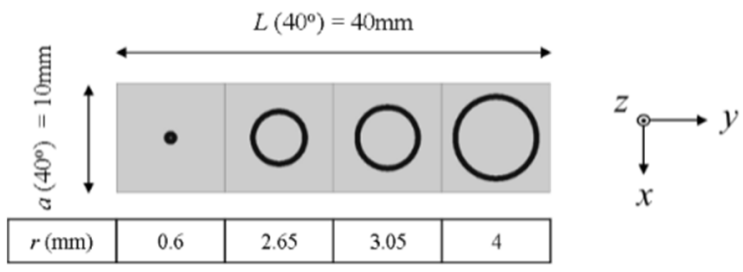

(b)

Fig. 2. Supercell of the proposed 1D PIRMS: (a) retrodirected angle of $20^{\circ}\left(\phi=90^{\circ}\right)$ and (b) retrodirected angle of $40^{\circ}(\phi=$ $90^{\circ}$.

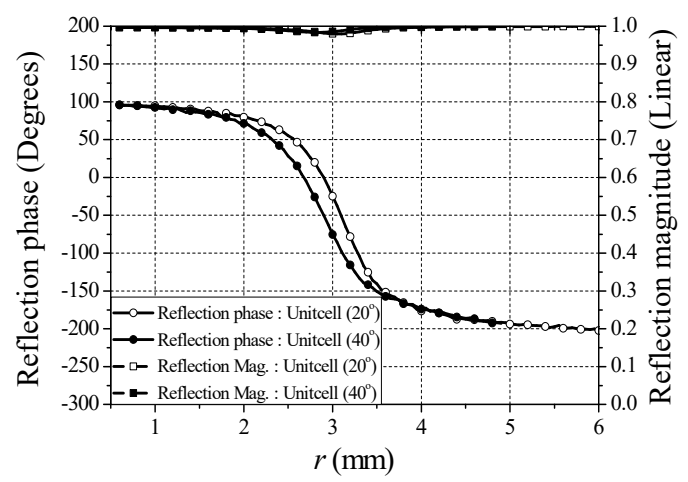

Fig. 3. Full-wave simulated reflection responses of the ring against the radius $(r)$ at $5.8 \mathrm{GHz}$. 


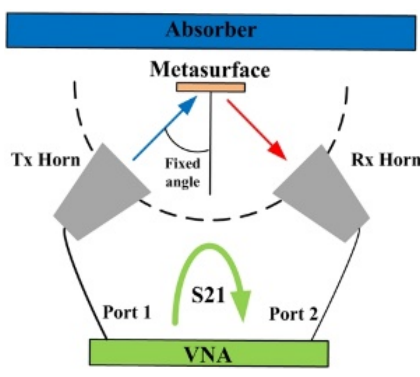

(a)

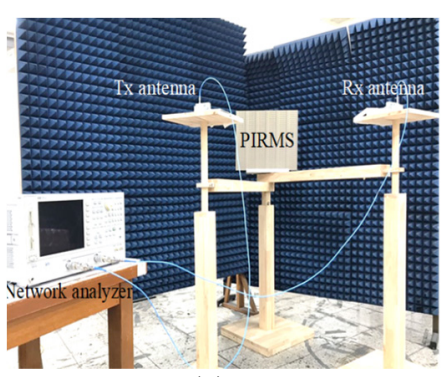

(b)
Fig. 4. (a) Schematic and (b) photograph of the measurement setup.

ured by fixing the transmitting horn antenna retro angle with the PIRMS and moving only the receiving horn antenna. To excite the plane wave, the distance between the antenna and PIRMS is selected to be $70 \mathrm{~cm}$, which satisfies the far-field radiation condition, as shown in Fig. 4. Moreover, the time gating function in the network analyzer is utilized to eliminate multiple reflections. Fig. 5(a) and (b) show a comparison in measured normalized reflected power between the copper and designed PIRMS at incident angle of $20^{\circ}$ and $40^{\circ}$, respectively. The gains of the utilized $\mathrm{Tx}$ and $\mathrm{Rx}$ antennas are equally approximately 11 $\mathrm{dBi}$. In addition, the $3 \mathrm{~dB}$ beamwidths of the antennas are $39^{\circ}$ and $40^{\circ}$ in the E-plane and $\mathrm{H}$-plane, respectively. Thus, the shape of the reflected power is slightly broad in the cases of

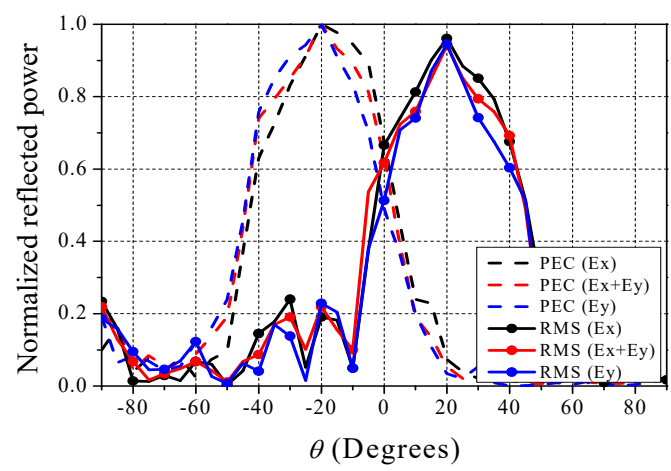

(a)

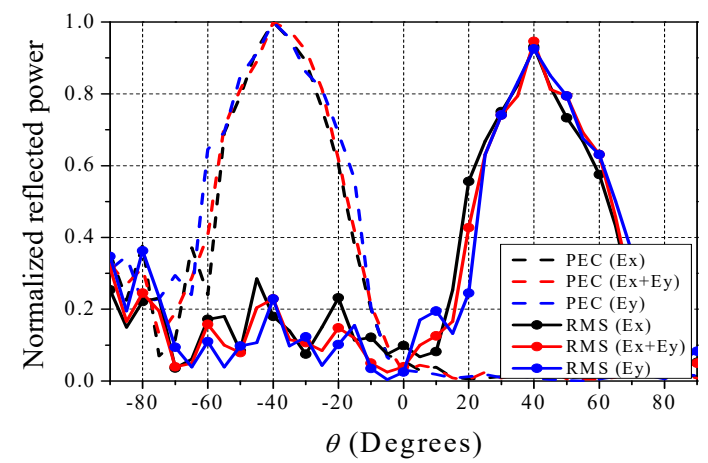

(b)

Fig. 5. Comparison of measured normalized reflected power between copper and proposed 1D PIRMS: (a) incident angle of $20^{\circ}$ (b) incident angle of $40^{\circ}$.
PEC and RMS. The measured retroreflected power efficiencies are more than $94 \%$ and $92 \%$ at the retrodirected angles of $20^{\circ}$ and $40^{\circ}$, respectively, regardless of the polarization.

\section{CONCLUSION}

In this letter, a 1D PIRMS is proposed using a ring structure on the ground plane. The 1D PIRMS is composed of supercells with the $2 \pi$ reflection phase gradient and the supercell is implemented by the series unitcells of a ring structure. To confirm retrodirectivity, two kinds of $1 \mathrm{D}$ PIRMSs, which are operated at two retrodirected angles of $20^{\circ}$ and $40^{\circ}$, were simulated and measured at $5.8 \mathrm{GHz}$. Based on the results, it can be concluded that the proposed 1D PIRMS perform with highly efficient retrodirectivity regardless of the polarization.

This research was supported by Basic Science Research Program through the National Research Foundation of Korea (NRF) funded by the Ministry of Education (No. 2015R1A6A1A03031833) and Ministry of Science, ICT and Future Planning, Korea, under the Information Technology Research Center support program (No. IITP-20172016-0-00291) supervised by the Institute for Information \& communications Technology Promotion (IITP).

\section{REFERENCES}

[1] N. Yu, P. Genevet, M. A. Kats, F. Aieta, J. P. Tetienne, F. Capasso, and Z. Gaburro, "Light propagation with phase discontinuities: generalized laws of reflection and refraction," Science, vol. 334, no. 6054, pp. 333-337, 2011.

[2] E. Doumanis, G. Goussetis, G. Papageorgiou, V. Fusco, R. Cahill, and D. Linton, "Design of engineered reflectors for radar cross section modification," IEEE Transactions on Antennas and Propagation, vol. 61, no. 1, pp. 232-239, 2013.

[3] N. M. Estakhri and A. Alu, "Wave-front transformation with gradient metasurfaces," Physical Review X, vol. 6, article no. 041008, 2016.

[4] T. V. Hoang and J. H. Lee, "Generation of multi-beam reflected from gradient-index metasurfaces," Results in Physics, vol. 10, pp. 424-426, 2018.

[5] M. Kalaagi and D. Seetharamdoo, "Retrodirective metasurface operating simultaneously at multiple incident angles," in Proceedings of 12th European Conference on Antennas and Propagation (EuCAP), London, UK, 2018, pp. 1-5.

[6] A. M. H. Wong, P. Christian, and G. V. Eleftheriades, "Binary Huygens' metasurfaces: experimental demonstration of simple and efficient near-grazing retroreflectors for TE and TM polarizations," IEEE Transactions on Antennas and Propagation, vol. 66, no. 6, pp. 2892-2903, 2018. 